\title{
Efectividade das infiltrações de corticosteróides nas doenças reumáticas peri-articulares: uma revisão baseada na evidência
}

Ana Rita Ferreira,* Eunice Carrapiço,* José Bravo Pimentão**

\section{RESUMO}

Introdução: As doenças reumáticas peri-articulares são muito prevalentes, têm um elevado peso económico e social e são causa frequente de consultas de medicina geral e familiar. As infiltrações de corticosteróides são importantes adjuvantes no tratamento das doenças reumáticas e podem ser realizadas pelo médico de família com as competências necessárias.

Objectivo: Rever a evidência sobre a efectividade das infiltrações de corticosteróides em várias doenças reumáticas peri-articulares. Fontes de dados: Cochrane Library, Best Practice, Clinical Evidence, Essential Evidence Plus e MEDLINE.

Métodos de revisão: Para cada uma das doenças seleccionadas para a revisão (síndroma do túnel cárpico, epicondilite, doença de De Quervain, dedo em gatilho, ombro doloroso, bursite trocantérica e bursite anserina) foi feita uma pesquisa sistemática de revisões sistemáticas, ensaios clínicos aleatorizados e controlados e normas de orientação clínica. As pesquisas foram realizadas em Março de 2011, utilizando como critérios de elegibilidade: (1) população de doentes com o diagnóstico; (2) intervenção com infiltração de corticosteróides versus ausência de tratamento, placebo ou outros tratamentos; (3) resultados de efectividade orientados para o doente; (4) data de publicação não anterior a 2000. Foram excluídos os estudos não acessíveis em texto integral.

Resultados: Foram incluídos 38 estudos. Na síndroma do túnel cárpico a infiltração é mais efectiva que o placebo. Na epicondilite a infiltração é benéfica na redução dos sintomas a curto-prazo. Na doença de De Quervain recomenda-se incluir a infiltração no tratamento inicial. No dedo em gatilho a infiltração é efectiva e aparentemente segura versus placebo. No ombro não é possível tirar conclusões firmes. Na bursite trocantérica existe uma recomendação fraca para a infiltração. Não foram obtidos resultados na bursite anserina.

Conclusão: As infiltrações de corticosteróides mostraram-se efectivas no tratamento de várias doenças reumáticas peri-articulares. No entanto, verificam-se diferenças consideráveis na magnitude e duração dos efeitos terapêuticos, o que invalida conclusões generalizadas.

Palavras-chave: Infiltrações; Corticosteróides; Efectividade; Doença Reumática; Periartrite.

\section{INTRODUÇÃO}

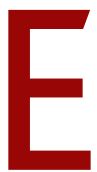

m Portugal as doenças reumáticas têm um elevado peso económico e social e são causa de 16-23\% das consultas em cuidados de saúde primários (CSP). ${ }^{1}$ Os sintomas músculo-esqueléticos traduzem, em muitos casos, lesões músculo-esqueléticas relacionadas com o trabalho (LMERT). ${ }^{2}$ As LMERT têm uma elevada prevalência em Portugal, identificando-se factores de risco de natureza ergonómica (como movimentos repetidos, levantamento e transporte de cargas), organizacional (como horas ou ritmos de trabalho excessivos) e individual (como o tabagismo e a obesidade)., ${ }^{1,2}$
As infiltrações músculo-esqueléticas são consideradas importantes adjuvantes na abordagem da doença articular e peri-articular, nomeadamente das LMERT., ${ }^{3,4}$ Actualmente, em Portugal, as infiltrações músculo-esqueléticas são maioritariamente realizadas em cuidados de saúde secundários (CSS) ou em medicina privada praticada por reumatologistas ou ortopedistas. Não obstante, vários autores consideram que as técnicas de infiltração músculo-esquelética poderão ser executadas de forma efectiva pelo médico

*Interna de Medicina Geral e Familiar USF Marginal (ACES Cascais)

**Médico responsável pelo Sector de Técnicas do Serviço de Reumatologia, Hospital Egas Moniz (Centro Hospitalar Lisboa Ocidental) 
de família, reconhecendo-se várias vantagens na realização destas técnicas em cuidados de saúde primários.,4

No contexto da implementação do projecto «Terapêutica por infiltração local com corticosteróides nas doenças reumáticas peri-articulares na USF Marginal», que teve como objectivo tornar disponível aos utentes esta opção de tratamento, foi feita uma revisão baseada na evidência sobre efectividade das infiltrações de corticosteróides em várias doenças reumáticas peri-articulares.

\section{MÉTODOS}

Foi realizada uma pesquisa sistemática entre 20 e 25 de Março de 2011, nas bases de dados Cochrane Library, Best Practice e Essential Evidence Plus, MEDLINE e Clinical Evidence, de revisões sistemáticas (RS), normas de orientação clínica (NOC) e ensaios clínicos aleatorizados e controlados (ECA) não incluídos nas RS, com data de publicação não anterior a 2000, utilizando os termos $M e S H$ «carpal tunnel syndrome», «tennis elbow», «De Quervain disease», «trigger finger», «shoulder pain», «trochanteric», «bursitis», «anserine» $\mathrm{e}$ «corticosteroid injection».

Utilizaram-se os seguintes critérios de elegibilidade: população de doentes com o diagnóstico de síndroma do túnel cárpico, epicondilite, doença de De Quervain, dedo em gatilho, ombro doloroso, bursite trocantérica ou bursite anserina, independentemente dos critérios de diagnóstico utilizados, idade, género e doenças associadas; intervenção terapêutica com infiltração peri-articular de corticosteróide (independentemente do corticosteróide, dose, técnica e associação a anestésico) versus ausência de tratamento, placebo ou outros tratamentos; resultados orientados para o doente (como alteração dos sintomas, função, qualidade de vida, satisfação e absentismo laboral); data de publicação não anterior a 2000.

Para os critérios de elegibilidade foram escolhidas as doenças reumáticas peri-articulares mais frequentes na população gerale em que a infiltração com corticosteróide seja utilizada como recurso terapêutico na prática clínica em Portugal. ${ }^{2}$

Para cada uma das doenças reumáticas peri-articulares que integram esta revisão foi feita uma pesquisa sistemática independente por um dos autores, o qual também foi responsável pela selecção e análise dos resultados obtidos. Os resumos foram seleccionados tendo em conta os critérios de inclusão, sendo posteriormente reunidos os artigos em texto integral referentes aos títulos e resumos seleccionados. Foram excluídos os estudos que não cumpriam os critérios de elegibilidade ou não acessíveis em texto integral.

Os resultados foram classificados de acordo com a taxonomia Strength of Recommendation Taxonomy (SORT). ${ }^{5}$ As NOC foram classificadas de acordo com a sua metodologia com base na classificação adoptada pelo Centro de Estudos de Medicina Baseada na Evidência da Faculdade de Medicina de Lisboa. ${ }^{6}$

\section{RESULTADOS}

Nas sete pesquisas sistemáticas realizadas independentemente para cada doença reumática peri-articular foram encontrados 515 resultados, dos quais foram selecionados 44 para leitura.

Destes, foram excluídas duas NOC, por não terem sido acessíveis em texto integral, dois ensaios clínicos, por não serem claros os processos de aleatorização e ocultação, e um ECA por não cumprir o critério de elegibilidade relativo à intervenção terapêutica. Foram, assim, incluídos 39 resultados (Quadro I), que correspondem a 36 artigos diferentes (porque três dos artigos foram resultado em mais do que uma revisão).

Quanto à síndroma do túnel cárpico (STC) (Quadro II), a infiltração local de corticosteróide é mais efectiva que o placebo após um mês [Força de Recomendação (FR) A] e parece promover uma maior melhoria clínica do que os corticosteróides orais até aos 3 meses (FR A) e do que uma injecção intramuscular de corticosteróide até 1 mês (FR B). ${ }^{7-12}$

Embora pareça haver uma tendência de redução ao longo do tempo, não se sabe com certeza quanto tempo dura o efeito benéfico da infiltração de corticosteróide e se há possibilidade de conseguir um alívio permanente. ${ }^{8}$

A revisão da Cochrane Database incluiu dois ECA que compararam a infiltração local de corticosteróide com a cirurgia no tratamento do STC. Estes estudos foram classificados como de boa qualidade nos parâmetros alocação, critérios de diagnóstico e diferenças de base, apresentando limitações na ocultação (não realizada ou inadequada). Os resultados não favorecem, de forma clara, uma intervenção sobre a outra, ${ }^{13}$ o que significa que não se pode concluir através da evidência actual sobre a efectividade da infiltração local de corticosteróide versus cirurgia no tratamento do STC, sendo necessários mais estudos. De salientar que, em termos de complicações do tratamento, estas poderão ser superiores no grupo da 


\begin{tabular}{|c|c|c|}
\hline Doenças reumáticas peri-articulares & N. ${ }^{\circ}$ Artigos encontrados & N. ${ }^{\circ}$ Artigos incluídos \\
\hline Síndroma do túnel cárpico & 64 & $6 \mathrm{RS}, 1 \mathrm{ECA}, 4 \mathrm{NOC}$ \\
\hline Epicondilite & 93 & $4 \mathrm{RS}, 1 \mathrm{ECA}, 3 \mathrm{NOC}$ \\
\hline Doença de De Quervain & 14 & $2 \mathrm{RS}, 1 \mathrm{ECA}, 1 \mathrm{NOC}$ \\
\hline Dedo em gatilho & 61 & $2 \mathrm{RS}, 2 \mathrm{ECA}, 1 \mathrm{NOC}$ \\
\hline Ombro doloroso & 160 & $5 \mathrm{RS}, 4 \mathrm{NOC}$ \\
\hline Bursite trocantérica & 70 & $2 \mathrm{NOC}$ \\
\hline Bursite anserina & 53 & 0 \\
\hline
\end{tabular}

e ausência de diferenças significativas às oito e doze semanas. Alguns ECA, de toxina contra placebo, têm mostrado benefício da toxina mas os resultados não foram consistentes. ${ }^{22}$

Quanto à efectividade da infiltração de corticosteróide na doença de $D e$ Quervain (Quadro IV), as duas RS incluídas basearam-se em apenas um ensaio clínico controlado que comparou infiltração de metilprednisolona com a utilização de tala em mulheres grávidas e puérperas. Este estudo mos- cirurgia comparativamente a tratamentos não cirúrgicos. ${ }^{13}$

As normas de orientação clínica incluídas nesta revisão sugerem a opção infiltração de corticosteróide, antes de considerar a cirurgia, se a doença for ligeira a moderada. $^{14-17}$

Em relação à epicondilite (Quadro III), existe forte evidência de que a infiltração de corticosteróide é benéfica na redução dos sintomas a curto-prazo, sendo claramente superior à ausência de tratamento, ao uso de anti-inflamatórios não esteróides (AINE), a tratamentos de medicina física e de reabilitação e ao uso de ortóteses (FR A)..$^{18-21}$

No entanto, vários ensaios clínicos de elevada qualidade e consistentes mostraram reversão dos efeitos com o tempo, sugerindo que a infiltração de corticosteróide é menos benéfica do que outros tratamentos conservadores a médio-longo prazo (FR A). ${ }^{18-22}$

A diferença entre os efeitos demonstrados a curto, médio e longo prazo contribui para explicar a inconsistência observada nas normas de orientação clínica. ${ }^{23-25}$

Na prática clínica, a infiltração de corticosteróide é frequentemente prescrita em combinação com anti-inflamatórios ou agentes físicos (fisioterapia), embora ainda não tenham sido demonstradas diferenças de efectividade com estas co-intervenções. Duas das RS incluídas realçam a necessidade de realizar estudos de tamanho suficiente para avaliar se os agentes físicos poderão reduzir a recorrência associada à infiltração de corticosteróide..$^{20,21}$

Tendo em conta que as tendinopatias têm escassa patogénese inflamatória, a investigação recente tem estudado a injecção de outras substâncias para além do corticosteróide. O ECA incluído sobre epicondilite comparou a injecção de toxina botulínica com a infiltração de corticosteróide e mostrou a superioridade deste às quatro semanas trou uma superioridade clara da infiltração de corticosteróide [Número Necessário de Tratar para Benefício (NNT)= 1] mas, sendo um estudo de baixa qualidade (com um número pequeno de participantes e apenas grávidas ou puérperas, pouco tempo de duração, limitações metodológicas), a aplicabilidade prática foi considerada limitada..$^{8,26}$

Posteriormente, foi publicado um ECA controlado que pretendeu estudar a efectividade da infiltração de corticosteróide contra placebo na doença de De Quervain, em CSP. Metodologicamente tratou-se de um ensaio de boa qualidade - em que os processos de aleatorização, alocação e ocultação, o seguimento, as características de base e as medições utilizadas foram adequados, apontando-se como limitação o facto de não ter sido atingido o número planeado de participantes. ${ }^{27}$

Este estudo mostrou que uma ou duas infiltrações de acetonido de triancinolona ${ }^{1} 10 \mathrm{mg} / \mathrm{ml}$, fornecidas pelo médico de família, causam uma maior melhoria sintomática do que o placebo (NNT=2). Os doentes que tiveram melhoria com o corticosteróide foram seguidos num estudo coorte de 12 meses que mostrou manutenção dos benefícios na maioria dos parâmetros avaliados. Assim, neste estudo sugere-se que a infiltração de corticosteróide seja utilizada como tratamento inicial na doença de De Quervain, referenciando-se para cirurgia em caso de resposta insufi-

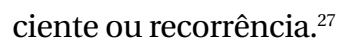

As sugestões clínicas apontadas por estes dois ECA são consistentes com a norma de orientação clínica incluída nesta revisão, que apoia a recomendação de tratamento conservador inicial, podendo utilizar-se a infiltração com

\footnotetext{
${ }^{1}$ Este corticosteróide é pouco utilizado na prática clínica por se considerar que é menos seguro que o acetato de metil-prednisolona.
} 
QUADRO II. Resultados sobre a efectividade da infiltração de corticosteróide no STC.

\begin{tabular}{|c|c|c|c|c|}
\hline $\begin{array}{l}\text { Tipo de artigo } \\
\text { Autor e ano }\end{array}$ & Título & Intervenções & Resultados & $\begin{array}{l}\text { NE/ } \\
\text { /FR }\end{array}$ \\
\hline $\begin{array}{l}\text { RS } \\
\text { Gerritsen } 2002^{7}\end{array}$ & $\begin{array}{l}\text { Conservative treatment options } \\
\text { for carpal tunnel syndrome: a } \\
\text { systematic review of randomised } \\
\text { controlled trials }\end{array}$ & $\begin{array}{l}3 \text { ECA }(n=129) \\
\text { Metilprednisolona } 40 \mathrm{mg} \\
\text { + lidocaína } 10 \mathrm{mg} ; \\
\text { metilprednisolona } 15 \mathrm{mg} ; \\
\text { betametasona } 1,5 \mathrm{mg} .\end{array}$ & $\begin{array}{l}\text { Há evidência limitada de que a } \\
\text { infiltração de corticosteróide é } \\
\text { mais efectiva do que o placebo } \\
\text { ou injecção IM de } \\
\text { corticosteróide após } 1 \text { mês. }\end{array}$ & 1 \\
\hline $\begin{array}{l}\text { RS } \\
\text { Agency for } \\
\text { Healthcare } \\
\text { Research and } \\
\text { Quality } 2002^{8}\end{array}$ & $\begin{array}{l}\text { Diagnosis and treatment of } \\
\text { worker related musculoskeletal } \\
\text { disorders of the upper extremity. } \\
\text { Evidence report/technology } \\
\text { assessment number } 62\end{array}$ & $\begin{array}{l}4 \text { ECA ( } n=261 \text { ) } \\
\text { Infiltração de corticosteróide } \\
\text { (vários) versus ausência de } \\
\text { tratamento, placebo e injecção IM } \\
\text { de corticosteróide. }\end{array}$ & $\begin{array}{l}\text { A infiltração levou a resultados } \\
\text { globais superiores. Não há } \\
\text { informação sobre diferenças entre } \\
\text { corticosteróides e sobre a duração } \\
\text { esperada de alívio. }\end{array}$ & 1 \\
\hline $\begin{array}{l}\text { RS } \\
\text { Piazzini } 2007^{9}\end{array}$ & $\begin{array}{l}\text { Systematic review of conservative } \\
\text { treatment of carpal tunnel } \\
\text { syndrome }\end{array}$ & $\begin{array}{l}7 \text { ECA ( } n=282 \text { ) } \\
\text { Infiltração de corticosteróide } \\
\text { (vários) versus outros tratamentos. }\end{array}$ & $\begin{array}{l}\text { Três estudos reportaram melhoria } \\
\text { significativa a curto-prazo versus } \\
\text { placebo e cinco melhoria } \\
\text { significativa desde o estado base. } \\
\text { Existe evidência forte da } \\
\text { efectividade dos corticosteróides } \\
\text { locais. }\end{array}$ & 1 \\
\hline $\begin{array}{l}\text { RS } \\
\text { Marshall } 2007^{10}\end{array}$ & $\begin{array}{l}\text { Local corticosteroid injection for } \\
\text { carpal tunnel syndrome }\end{array}$ & $\begin{array}{l}2 \text { ECA ( } n=141 \text { ) infiltração versus } \\
\text { placebo; } 2 \text { ECA ( } n=97 \text { ) infiltração } \\
\text { versus corticosteróide oral; } 1 \text { ECA } \\
\text { ( } n=23 \text { ) infiltração versus AINE e } \\
\text { tala; } 1 \text { ECA ( } n=40 \text { ) infiltração } \\
\text { versus laser Helium-Neon; } 2 \text { ECA } \\
\text { ( } n=51 \text { ) infiltração versus } \\
\text { iontoforese; } 2 \text { ECA ( } n=22 \text { ) } \\
\text { infiltração versus fonoforese. }\end{array}$ & $\begin{array}{l}\text { A infiltração de corticosteróide } \\
\text { provoca uma maior melhoria } \\
\text { clínica do que placebo até } 1 \text { mês } \\
\text { e do que corticosteróides orais } \\
\text { até } 3 \text { meses após a técnica. A } \\
\text { infiltração não melhora } \\
\text { significativamente os resultados } \\
\text { versus AINE ou tala às } 6 \text { semanas } \\
\text { ou laser aos } 6 \text { meses. }\end{array}$ & 1 \\
\hline $\begin{array}{l}\text { RS } \\
\text { Asworth } 2010^{11}\end{array}$ & Carpal tunnel syndrome & $\begin{array}{l}3 \text { ECA ( } n=173 \text { ) infiltração versus } \\
\text { placebo às } 2-6 \text { semanas; } 2 \text { ECA } \\
\text { ( } n=97 \text { ) infiltração versus } \\
\text { corticosteróides sistémicos às } \\
4-12 \text { semanas; } 2 \text { ECA ( } n=151 \text { ) } \\
\text { infiltração versus cirugia. }\end{array}$ & $\begin{array}{l}\text { Versus placebo e corticosteróides } \\
\text { sistémicos, a infiltração de } \\
\text { corticosteróide parece mais } \\
\text { efectiva a melhorar os sintomas. } \\
\text { Versus cirurgia, não se sabe se a } \\
\text { infiltração é mais efectiva a } \\
\text { melhorar os sintomas. Versus } \\
\text { cirurgia de libertação, pode ser } \\
\text { igualmente efectiva a melhorar a } \\
\text { função da mão. }\end{array}$ & 1 \\
\hline
\end{tabular}

corticosteróide para além da evicção de movimentos provocadores de dor e a imobilização com tala como forma de tratar os sintomas da doença de De Quervain (FR B). ${ }^{28}$

Em relação ao tratamento do dedo em gatilho no adulto (QuadroV), a revisão da Cochrane Database inclui dois ensaios clínicos sobre infiltração de corticosteróide com lidocaína versus infiltração de lidocaína que, apesar da baixa qualidade metodológica, têm resultados consistentes no sentido da efectividade da infiltração de corticosteróide, com efeitos terapêuticos de dimensão considerável às quatro semanas $(\mathrm{NNT}=3)$ e mantidos aos quatro meses. ${ }^{29}$ As implicações práticas destes resultados 


\begin{tabular}{|c|c|c|c|c|}
\hline $\begin{array}{l}\text { Tipo de artigo } \\
\text { Autor e ano }\end{array}$ & Título & Intervenções & Resultados & $\begin{array}{l}\mathrm{NE} / \\
\text { /FR }\end{array}$ \\
\hline $\begin{array}{l}\text { ECA } \\
\text { Peters-Velutha- } \\
\text { maningal } \\
2010^{12}\end{array}$ & $\begin{array}{l}\text { Randomised controlled trial of } \\
\text { local corticosteroid injections for } \\
\text { carpal tunnel syndrome in general } \\
\text { practice }\end{array}$ & $\begin{array}{l}1 \text { ECA duplamente cego ( } \mathrm{n}=35 / 36 \\
\text { e } \mathrm{n}=31 / 33 \text { ) Infiltração de } 1 \mathrm{ml} \\
\text { acetonido de triamcinolona } \\
10 \mathrm{mg} / \mathrm{ml} \text { (TCA) versus } 1 \mathrm{ml} \mathrm{NaCl} \\
\text { por clínicos gerais. } \\
\text { Avaliação aos } 7 \text { dias da resposta } \\
\text { directa ao tratamento, melhoria } \\
\text { percepcionada e gravidade de } \\
\text { sintomas pela Symptom Severity } \\
\text { Scale. }\end{array}$ & $\begin{array}{l}\text { O grupo TCA teve melhores } \\
\text { resultados com um número } \\
\text { necessário para tratar (NNT) para } \\
\text { obter resposta satisfatória parcial } \\
\text { ou resolução completa de } 3.0 \\
\text { estudo coorte mostrou que os } \\
\text { benefícios se deteriorara no } \\
\text { seguimento de } 12 \text { meses - } \\
\text { metade do grupo que respondeu } \\
\text { teve recorrências. }\end{array}$ & 1 \\
\hline $\begin{array}{l}\text { RS } \\
\text { Verdugo } 2008^{13}\end{array}$ & $\begin{array}{l}\text { Surgical versus non-surgical } \\
\text { treatment for carpal tunnel } \\
\text { syndrome }\end{array}$ & $\begin{array}{l}2 \text { Ensaios clínicos }(n=151) \\
\text { Infiltração de corticosteróide } \\
\text { versus cirurgia. }\end{array}$ & $\begin{array}{l}\text { Os resultados combinados não } \\
\text { favorecem claramente uma } \\
\text { intervenção sobre a outra, pelo } \\
\text { menos a curto prazo. }\end{array}$ & 2 \\
\hline $\begin{array}{l}\text { Normas da } \\
\text { melhor práctica } \\
\text { Work Loss Data } \\
\text { Institute - Public } \\
\text { For Profit } \\
\text { Organization } \\
2003^{14}\end{array}$ & $\begin{array}{l}\text { Carpal tunnel syndrome (acute \& } \\
\text { chronic) }\end{array}$ & \multicolumn{2}{|c|}{$\begin{array}{l}\text { Na doença ligeira a moderada ( } 70 \% \text { dos casos) recomenda-se } \\
\text { terapêutica conservadora inicial. Prescrever modificação da actividade. } \\
\text { Se não melhorar prescrever tratamento físico ou ocupacional. Se não } \\
\text { melhorar tentar infiltração de corticosteróide (grande probabilidade } \\
\text { de melhoria mas pode haver recorrência dos sintomas dentro de } \\
\text { alguns meses; a melhoria inicial pode ser um bom indicador do } \\
\text { sucesso da cirurgia). Deve ser realizada por um médico treinado. } \\
\text { Recomenda-se apenas uma infiltração. }\end{array}$} & nd* \\
\hline $\begin{array}{l}\text { Normas } \\
\text { baseadas na } \\
\text { evidência } \\
\text { German } \\
\text { Societies of } \\
\text { Handsurgery } \\
\text { (...) } 2007^{15}\end{array}$ & $\begin{array}{l}\text { Diagnosis and therapy of carpal } \\
\text { tunnel syndrome }\end{array}$ & \multicolumn{2}{|c|}{$\begin{array}{l}\text { Entre os métodos conservadores, o tratamento com a tala e a } \\
\text { infiltração de corticosteróides são eficazes. Os corticosteróides orais, } \\
\text { imobilização e ultrasons mostraram apenas benefício a curto prazo. } \\
\text { O tratamento cirúrgico é claramente superior aos outros métodos. }\end{array}$} & nd* \\
\hline \multirow{3}{*}{$\begin{array}{l}\text { Normas } \\
\text { baseadas na } \\
\text { evidência } \\
\text { AAOS } 2008^{16}\end{array}$} & \multirow[t]{3}{*}{$\begin{array}{l}\text { Treatment of carpal tunnel } \\
\text { syndrome }\end{array}$} & \multicolumn{2}{|c|}{$\begin{array}{l}\text { Um curso de tratamento não-cirúrgico é uma opção. A cirurgia inicial } \\
\text { é uma opção se houver evidência clínica de desnervação ou se o } \\
\text { doente preferir. }\end{array}$} & $\mathrm{C}$ \\
\hline & & \multicolumn{2}{|c|}{$\begin{array}{l}\text { Sugerimos outro tratamento não-cirúrgico ou cirurgia quando o } \\
\text { actual tratamento não resolve os sintomas até } 2-7 \text { semanas. }\end{array}$} & B \\
\hline & & \multicolumn{2}{|c|}{$\begin{array}{l}\text { A infiltração de corticosteróide ou o uso de tala é sugerida antes de } \\
\text { considerar a cirurgia. }\end{array}$} & B \\
\hline \multirow{3}{*}{$\begin{array}{l}\text { Normas da } \\
\text { melhor prática } \\
\text { EBM Guidelines } \\
2009^{17}\end{array}$} & \multirow[t]{3}{*}{ Carpal tunnel syndrome } & \multicolumn{2}{|c|}{$\begin{array}{l}\text { As modalidades de terapêutica conservadora (por ex. tala) são } \\
\text { preferíveis. }\end{array}$} & $C$ \\
\hline & & \multicolumn{2}{|c|}{$\begin{array}{l}\text { Os sintomas frequentemente desvanecem-se quando os factores } \\
\text { desencadeantes são resolvidos. }\end{array}$} & $C$ \\
\hline & & \multicolumn{2}{|c|}{ A terapêutica com infiltração pode ser tentada antes da cirurgia. } & $A$ \\
\hline
\end{tabular}

*Não definido 
QUADRO III. Resultados sobre a efectividade da infiltração de corticosteróide na epicondilite.

\begin{tabular}{|c|c|c|c|c|}
\hline $\begin{array}{l}\text { Tipo de artigo } \\
\text { Autor e ano }\end{array}$ & Título & Intervenções & Resultados & $\begin{array}{l}\text { NE/ } \\
\text { /FR }\end{array}$ \\
\hline $\begin{array}{l}\text { RS } \\
\text { Smidt } 2002^{18}\end{array}$ & $\begin{array}{l}\text { Corticosteroid injections for } \\
\text { lateral epicondylitis: a systematic } \\
\text { review }\end{array}$ & $\begin{array}{l}13 \text { ECA; } 15 \text { comparações }(\mathrm{n}=1028) \\
\text { Infiltração de corticosteróides } \\
\text { versus placebo, anestésico local, } \\
\text { outras infiltrações de } \\
\text { corticosteróides ou outros } \\
\text { tratamentos conservadores. } \\
\text { Avaliação da melhoria global e } \\
\text { força de preensão. }\end{array}$ & $\begin{array}{l}13 \text { comparações mostraram } \\
\text { superioridade da infiltração a } \\
\text { curto prazo (<6 semanas) que foi } \\
\text { estatística e clinicamente } \\
\text { relevante. A médio-longo prazo } \\
\text { (>6 meses) os resultados não se } \\
\text { mantiveram. } \\
\text { A evidência é insuficiente para } \\
\text { suportar ou refutar a efectividade } \\
\text { da infiltração. }\end{array}$ & 2 \\
\hline $\begin{array}{l}\text { RS } \\
\text { Buchbinder } \\
2008^{19}\end{array}$ & Tennis elbow & $\begin{array}{l}2 \text { RS; } 3 \text { ECA } \\
\text { Infiltração de corticosteróide } \\
\text { versus placebo ou ausência de } \\
\text { tratamento, anestésico local, } \\
\text { ortóteses, versus exercício e } \\
\text { imobilização ou AINE oral; } \\
\text { Avaliação do alívio da dor, } \\
\text { melhoria global e melhoria } \\
\text { funcional. }\end{array}$ & $\begin{array}{l}\text { Alívio da dor } \\
\text { - Pode reduzir mais a dor do que } \\
\text { placebo / ausência de } \\
\text { tratamento (às } 8 \text { semanas e } 6 \\
\text { meses), exercício mais } \\
\text { imobilização (às } 6 \text { semanas mas } \\
\text { não às } 52 \text { semanas) ou terapia } \\
\text { de ondas de choque } \\
\text { extracorpórea (às } 6 \text { semanas e } \\
3 \text { meses). } \\
\text { - Versus AINE, desconhece-se se } \\
\text { alivia mais a dor às } 4 \text { semanas, } \\
\text { podendo ser pior às } 26 \text { semanas. } \\
\text { Melhoria global } \\
\text { - Parece ser melhor a curto- } \\
\text {-prazo do que os outros } \\
\text { tratamentos. } \\
\text { Melhoria funcional } \\
\text { - Melhor do que placebo/ } \\
\text { /ausência de tratamento a curto } \\
\text { prazo mas não a longo-termo. } \\
\text { - Não se sabe se é superior à } \\
\text { fisioterapia. }\end{array}$ & 2 \\
\hline
\end{tabular}

são limitadas pela qualidade dos estudos, pela realização em CSS, pelo limite temporal de quatro meses e pela inexistência de ECA que comparem directamente a infiltração de corticosteróide com cirurgia ou tala à data da revisão. Não obstante, sendo uma modalidade de tratamento fácil de aplicar, com menos custos e menos invasiva que a cirurgia, os autores consideram que possa ser considerada em primeira linha. ${ }^{29}$

A segunda revisão sistemática sobre dedo em gatilho incluiu, para além dos dois ensaios clínicos já referidos, outros dois estudos: um sobre a infiltração de corticosteróide versus cirurgia de libertação percutânea com a infiltração de corticosteróide (sucesso terapêutico $47 \%$ versus $91 \%$ ) e um outro sobre corticosteróide intra-tendinoso versus corticosteróide subcutâneo (sem diferenças significativas nos resultados). Devido às limitações metodológicas da revisão os resultados devem ser interpretados cuidadosamente. ${ }^{30}$ 


\section{QUADRO III. (continuação)}

\begin{tabular}{|c|c|c|c|c|}
\hline $\begin{array}{l}\text { Tipo de artigo } \\
\text { Autor e ano }\end{array}$ & Título & Intervenções & Resultados & $\begin{array}{l}\text { NE/ } \\
\text { /FR }\end{array}$ \\
\hline $\begin{array}{l}\text { RS } \\
\text { Barr } 2009^{20}\end{array}$ & $\begin{array}{l}\text { Effectiveness of corticosteroid } \\
\text { injections compared with } \\
\text { physiotherapeutic interventions } \\
\text { for lateral epicondylitis }\end{array}$ & $5 \mathrm{ECA}(n=597)$ & $\begin{array}{l}\text { As infiltrações mostraram uma } \\
\text { maior efectividade do que a } \\
\text { fisioterapia a curto prazo (efeitos } \\
\text { de elevada dimensão) mas as } \\
\text { recorrências variaram entre } \\
\text { 34-72\%. A fisioterapia mostrou } \\
\text { maior efectividade que a infiltração } \\
\text { a médio-longo prazo (efeitos de } \\
\text { dimensão moderada a elevada). } \\
\text { Poucos estudos e de baixa qualidade } \\
\text { avaliaram os benefícios de combinar } \\
\text { a infiltração com fisioterapia, sendo } \\
\text { uma área importante para a futura } \\
\text { investigação. }\end{array}$ & 1 \\
\hline $\begin{array}{l}\text { RS } \\
\text { Coombes } \\
2010^{21}\end{array}$ & $\begin{array}{l}\text { Efficacy and safety of } \\
\text { corticosteroid injections and other } \\
\text { injections for management of } \\
\text { tendinopathy: a systematic review } \\
\text { of randomised controlled trials. }\end{array}$ & 12 ECA; 18 análises $(n=1171)$ & $\begin{array}{l}\text { Evidência forte mostra que a } \\
\text { infiltração é benéfica a curto-prazo } \\
\text { e superior a ausência de intervenção, } \\
\text { AINE, e fisioterapia. Não obstante, } \\
\text { evidência forte sugere também que } \\
\text { estes efeitos revertem a médio- } \\
\text {-longo prazo, sendo a infiltração } \\
\text { menos benéfica que outras opções } \\
\text { após } 26 \text { semanas. } \\
\text { Os estudos que compararam } \\
\text { infiltração com fisioterapia, } \\
\text { embora consistentes, têm } \\
\text { heterogeneidade significativa. }\end{array}$ & 1 \\
\hline $\begin{array}{l}\text { ECA } \\
\text { Lin } 2010^{22}\end{array}$ & $\begin{array}{l}\text { Comparison between botulinum } \\
\text { toxin and corticosteroid injection } \\
\text { in the treatment of acute and } \\
\text { subacute tennis elbow: a } \\
\text { prospective, randomized, } \\
\text { double-blind, active drug- } \\
\text {-controlled pilot study }\end{array}$ & $\begin{array}{l}1 \text { ECA duplamente cego }(n=16) \\
\text { Injecção de toxina botulínica A } \\
\text { versus injecção de acetonido de } \\
\text { triamcinolona (TCA). } \\
\text { Avaliação da percepção da dor, da } \\
\text { força de preensão e da qualidade } \\
\text { de vida às } 4,8 \text { e } 12 \text { semanas. }\end{array}$ & $\begin{array}{l}\text { Às } 4 \text { semanas a redução da dor foi } \\
\text { significativamente maior no grupo } \\
\text { TCA. } \\
\text { Houve uma diminuição da força } \\
\text { de preensão no grupo da toxina } \\
\text { às } 4 \text { semanas. } \\
\text { Às } 8 \text { e às } 12 \text { semanas as diferenças } \\
\text { não foram estatisticamente } \\
\text { significativas. }\end{array}$ & 2 \\
\hline $\begin{array}{l}\text { Normas da } \\
\text { melhor prática } \\
\text { EBM Gulidelines } \\
2008^{23}\end{array}$ & $\begin{array}{l}\text { Lateral and medial epicondylitis } \\
\text { of elbow }\end{array}$ & \multicolumn{2}{|c|}{$\begin{array}{l}\text { As infiltrações locais com corticosteróides podem ser apropriadas } \\
\text { como tratamento inicial, especialmente se a dor for moderada a grave. }\end{array}$} & nd \\
\hline $\begin{array}{l}\text { Normas da } \\
\text { melhor prática } \\
\text { Work Loss Data } \\
\text { Institute } 2008^{24}\end{array}$ & Elbow (acute \& chronic) & \multicolumn{2}{|c|}{$\begin{array}{l}\text { As infiltrações não são recomendadas por rotina porque podem } \\
\text { aumentar a recorrência da dor nos 3-12 meses de seguimento, apesar } \\
\text { de causarem um bom alívio da dor a curto prazo (< } 6 \text { semanas). }\end{array}$} & A \\
\hline
\end{tabular}




\section{QUADRO III. (continuação)}

\begin{tabular}{|c|c|c|c|c|}
\hline $\begin{array}{l}\text { Tipo de artigo } \\
\text { Autor e ano }\end{array}$ & Título & Intervenções & Resultados & $\begin{array}{l}\text { NE/ } \\
\text { /FR }\end{array}$ \\
\hline $\begin{array}{l}\text { Normas basea- } \\
\text { das na evidência } \\
\text { American } \\
\text { College of } \\
\text { Occupational } \\
\text { and Environ- }\end{array}$ & Elbow disorders & \multicolumn{2}{|c|}{$\begin{array}{l}\text { As infiltrações locais de corticosteróide para a epicondilite e } \\
\text { epitrocleíte têm evidência de eficácia a curto-prazo, não tendo sido } \\
\text { demonstrada eficácia a longo prazo. Considerar a terapêutica com } \\
\text { infiltração apenas se o tratamento conservador falhar após 3-4 } \\
\text { semanas. }\end{array}$} & $B$ \\
\hline $\begin{array}{l}\text { mental Medicine } \\
2007^{25}\end{array}$ & & \multicolumn{2}{|c|}{$\begin{array}{l}\text { Bupivacaína é superior à lidocaína quando combinada com o } \\
\text { corticosteróide na infiltração. }\end{array}$} & $B$ \\
\hline
\end{tabular}

\section{QUADRO IV. Resultados sobre a efectividade da infiltração de corticosteróide na tenossinovite de De Quervain.}

\begin{tabular}{|c|c|c|c|c|}
\hline $\begin{array}{l}\text { Tipo de artigo } \\
\text { Autor e ano }\end{array}$ & Título & Intervenções & Resultados & $\begin{array}{l}\mathrm{NE} / \\
\text { /FR }\end{array}$ \\
\hline $\begin{array}{l}\text { RS } \\
\text { Peters-Velutha- } \\
\text { maningal } \\
2009^{26}\end{array}$ & $\begin{array}{l}\text { Corticosteroid injection for de } \\
\text { Quervain's tenosynovitis }\end{array}$ & $\begin{array}{l}1 \text { Ensaio clínico controlado, } \\
\text { pseudo-aleatorizado e não cego } \\
(\mathrm{n}=18 \text { ) } \\
\text { Inclui grávidas e puérperas } \\
\text { submetidas infiltração de metil- } \\
\text {-prednisolona e bupivacaína } \\
\text { versus imobilização do } 1^{\circ} \text { dedo } \\
\text { após } 1-6 \text { dias. }\end{array}$ & $\begin{array}{l}\text { 9/9 no grupo da infiltração } \\
\text { tiveram alívio completo da dor } \\
\text { versus } 0 / 9 \text { no grupo da } \\
\text { imobilização; número necessário } \\
\text { para tratar para benefício (NNTB) } \\
\text { de } 1 ; \text { IC } 95 \% 0,8-1,2 \text {. } \\
\text { Não foram observados efeitos } \\
\text { adversos ou complicações. } \\
\text { Apesar da evidência prata* de } \\
\text { efectividade da infiltração versus } \\
\text { tala, a aplicabilidade dos resultados } \\
\text { à prática é limitada porque o ECA } \\
\text { tinha uma amostra pequena, } \\
\text { apenas incluiu grávidas e puérperas } \\
\text { e teve uma baixa qualidade } \\
\text { metodológica. }\end{array}$ & 2 \\
\hline $\begin{array}{l}\text { RS } \\
\text { Agency for } \\
\text { Healthcare } \\
\text { Research and } \\
\text { Quality. } 2002^{8}\end{array}$ & $\begin{array}{l}\text { Diagnosis and treatment of worker } \\
\text { related musculoskeletal disorders } \\
\text { of the upper extremity. Evidence } \\
\text { report/technology assessment } \\
\text { number } 62\end{array}$ & 1 Ensaio clínico não aleatorizado & $\begin{array}{l}\text { Apesar de um ensaio não } \\
\text { aleatorizado ter mostrado que a } \\
\text { infiltração de corticosteróide mais } \\
\text { lidocaína produziu um maior } \\
\text { sucesso terapêutico que a } \\
\text { imobilização, houve problemas de } \\
\text { desenho do estudo pelo que é } \\
\text { difícil obter conclusões. }\end{array}$ & 2 \\
\hline
\end{tabular}

Posteriormente à publicação das duas RS, foi publicado um ECA de boa qualidade, realizado em CSP, que mostrou efectividade superior da infiltração de corticosteróide contra placebo (com NNT=4) a curto prazo e manutenção dos efeitos benéficos durante o período de seguimento de 12 meses, sem efeitos adversos importantes. ${ }^{31}$

Assim, pode concluir-se que existe evidência de boa qualidade proveniente de um ECA (consistente com estudos de 


\begin{tabular}{|c|c|c|c|c|}
\hline $\begin{array}{l}\text { Tipo de artigo } \\
\text { Autor e ano }\end{array}$ & Título & Intervenções & Resultados & $\begin{array}{l}\text { NE/ } \\
\text { /FR }\end{array}$ \\
\hline $\begin{array}{l}\text { ECA } \\
\text { Peters-Velutha- } \\
\text { maningal } \\
2009^{27}\end{array}$ & $\begin{array}{l}\text { Randomised controlled trial of } \\
\text { local corticosteroid injections for } \\
\text { de Quervain's tenosynovitis in } \\
\text { general practice }\end{array}$ & $\begin{array}{l}1 \text { ECA duplamente cego }(\mathrm{n}=21 \text { ) } \\
\text { Infiltrações de TCA versus placebo. } \\
\text { Avaliação da efectividade a curto } \\
\text { prazo (resposta directa ao } \\
\text { tratamento, intensidade da dor, } \\
\text { estado funcional [Dutch AIMS-2- } \\
\text {-HFF] e melhoria percepcionada). } \\
1 \text { Estudo prospectivo aberto }(n=12) \\
\text { Avaliação da efectividade a } \\
\text { longo-prazo. }\end{array}$ & $\begin{array}{l}\text { A curto-prazo a infiltração teve } \\
\text { melhores resultados que o placebo } \\
(p<0,05) \text { na resposta directa ao } \\
\text { tratamento ( } 78 \% \text { versus } 25 \% \text {;), } \\
\text { na melhoria percepcionada ( } 78 \% \\
\text { versus } 33 \%) \text { e no alívio da dor } \\
(4,27 \text { versus } 1,33) \text {; NNT=2. Na } \\
\text { melhoria funcional medida os } \\
\text { resultados foram semelhantes } \\
\text { nos dois grupos. } \\
\text { Os efeitos benéficos da infiltração } \\
\text { mantiveram-se a longo-prazo ( } 12 \\
\text { meses) quanto a melhoria da dor } \\
\text { e pontuações do Dutch AIMS-2- } \\
\text {-HFF mas não quanto à melhoria } \\
\text { percepcionada. Não foram } \\
\text { observados efeitos adversos. }\end{array}$ & 2 \\
\hline $\begin{array}{l}\text { Normas da } \\
\text { melhor prática } \\
\text { EBM Guidelines } \\
2009^{28}\end{array}$ & $\begin{array}{l}\text { De Quervain's disease and other } \\
\text { tendinitides of the wrist and } \\
\text { forearm }\end{array}$ & \multicolumn{2}{|c|}{$\begin{array}{l}\text { Avaliar a tensão da mão e reduzi-la. Os movimentos que provocam } \\
\text { sintomas devem ser evitados. Pode ser utilizada uma tala como } \\
\text { suporte se necessário. } \\
\text { Se estas medidas falharem pode ser tentada uma infiltração, } \\
\text { principalmente na tenossinovite estenosante. }\end{array}$} & $n d^{* *}$ \\
\hline
\end{tabular}

*A Cochrane MusculoskeletalGroup propõe classificar a força da evidência nos seguintes graus (por ordem decrescente de força): platina, ouro, prata, bronze

**Não definido

baixa qualidade anteriores) de que a infiltração de corticosteróide é efectiva e aparentemente segura no tratamento do dedo em gatilho quando comparada com placebo (FR B), sendo proposta como tratamento de primeira linha. ${ }^{31,32}$

De notar que um ECA incluído nesta revisão mostrou que a efectividade das infiltrações parece ser menor na subpopulação dos doentes diabéticos (principalmente naqueles com manifestações sistémicas), sendo mais provável que estes necessitem de cirurgia. ${ }^{33}$

Em relação à efectividade da infiltração de corticosteróide no ombro doloroso (Quadro VI), com base nos estudos seleccionados não é possível tirar conclusões firmes sobre o benefício a curto ou longo prazo da infiltração. Há pouca evidência para suportar ou refutar a efectividade deste tratamento. ${ }^{34-41}$

Desconhece-se se a infiltração com corticosteróide é mais efectiva do que os AINE ou os tratamentos de me- dicina física e de reabilitação. ${ }^{21,34,36,37}$

As infiltrações subacromiais com corticosteróide podem ajudar a controlar a dor, facilitando os tratamentos de medicina física e de reabilitação. ${ }^{41}$

Na periartrite calcificante a infiltração no ponto subacromial poderá ser considerada como tratamento de primeira linha. ${ }^{39}$

A infiltração poderá ser repetida uma a três vezes em intervalos não inferiores a três semanas..$^{39,40}$

Em relação à efectividade da infiltração de corticosteróide na bursite trocantérica (Quadro VII), foram incluídas apenas duas NOC. ${ }^{41,42}$ Ambas consideram que a infiltração com corticosteróide e anestésico é uma opção terapêutica útil, que apresenta frequentemente bons resultados. Uma das NOC recomenda a infiltração como tratamento de escolha para a dor trocantérica. ${ }^{41}$

Quanto à revisão da efectividade da infiltração com cor- 
QUADRO V. Resultados sobre a efectividade da infiltração de corticosteróide no dedo em gatilho.

\begin{tabular}{|c|c|c|c|c|}
\hline $\begin{array}{l}\text { Tipo de artigo } \\
\text { Autor e ano }\end{array}$ & Título & Intervenções & Resultados & $\begin{array}{l}\text { NE/ } \\
\text { /FR }\end{array}$ \\
\hline $\begin{array}{l}\text { RS } \\
\text { Peters-Velutha- } \\
\text { maningal } \\
2009^{29}\end{array}$ & $\begin{array}{l}\text { Corticosteroid injection for trigger } \\
\text { finger in adults }\end{array}$ & $\begin{array}{l}2 \text { Ensaios clínicos controlados de } \\
\text { baixa qualidade }(n=63) \\
\text { Realizados em Cuidados de Saúde } \\
\text { Secundários com resultados } \\
\text { consistentes. }\end{array}$ & $\begin{array}{l}\text { Às } 4 \text { semanas as infiltrações de } \\
\text { corticosteroide e lidocaína são } \\
\text { mais efectivas do que infiltrações } \\
\text { de lidocaína com RR de } 3,15 \text { e } \\
\text { NNT de } 3 \text {. Um estudo mostrou } \\
\text { que os efeitos dos corticosteróides } \\
\text { mantinham-se aos } 4 \text { meses. } \\
\text { Não foram registados efeitos } \\
\text { adversos. } \\
\text { Os autores concluem que existe } \\
\text { evidência prata de que as } \\
\text { infiltrações de corticosteróides } \\
\text { são efectivas. Sugerem este } \\
\text { tratamento como 1. }{ }^{\text {la linha. }}\end{array}$ & 2 \\
\hline $\begin{array}{l}\text { RS } \\
\text { Fleisch } 2007^{30}\end{array}$ & $\begin{array}{l}\text { Corticosteroid injections in the } \\
\text { treatment of trigger finger: a level } \\
\text { I and II systematic review }\end{array}$ & $4 \mathrm{ECA}(n=285)$ & $\begin{array}{l}\text { No geral, } 57 \% \text { dos doentes tratados } \\
\text { com infiltração de corticosteróide } \\
\text { responderam ao tratamento. } \\
\text { As infiltrações foram significativa- } \\
\text { mente mais efectivas que o } \\
\text { placebo em dois estudos: } 64 \% \\
\text { versus } 20 \%(p<0,02) \text { e } 60 \% \\
\text { versus } 15 \% \text { ( } p<0,05) \text {. } \\
\text { A infiltração com a libertação } \\
\text { percutânea alivia a dor em } 91 \% \\
\text { dos doentes versus } 47 \% \text { nos } \\
\text { doentes com infiltração isolada } \\
(p=0,001) \text {. }\end{array}$ & 2 \\
\hline $\begin{array}{l}\text { ECA } \\
\text { Peters-Velutha- } \\
\text { maningal } \\
2008^{31}\end{array}$ & $\begin{array}{l}\text { Corticosteroid injections effective } \\
\text { for trigger finger in adults in } \\
\text { general practice: a double-blinded } \\
\text { randomised placebo controlled } \\
\text { trial }\end{array}$ & $\begin{array}{l}1 \text { ECA duplamente cego }(n=50) \\
\text { Uma a duas infiltrações de TCA } \\
\text { versus } \mathrm{NaCl} \text {. } \\
\text { Avaliação a curto-prazo ( } 1 \text { semana) } \\
\text { da resposta directa ao tratamento, } \\
\text { melhoria percepcionada, dor e } \\
\text { melhoria funcional. }\end{array}$ & $\begin{array}{l}\text { A curto prazo as infiltrações foram } \\
\text { significativamente mais efectivas } \\
\text { (RR=0,38; NNT=4). } \\
\text { Os benefícios mantiveram-se } \\
\text { durante os } 12 \text { meses de seguimento. } \\
\text { Não se observaram efeitos adversos. } \\
\text { Uma ou duas infiltrações de TCA } \\
10 \text { mg/ml é um método de } \\
\text { tratamento efectivo e seguro. } \\
\text { Sugere-se que constitua o } \\
\text { tratamento inicial, recorrendo-se } \\
\text { à cirurgia em caso de resposta } \\
\text { insuficiente ou recorrência. }\end{array}$ & 1 \\
\hline $\begin{array}{l}\text { Normas da } \\
\text { melhor prática } \\
\text { EBM } \\
\text { Guidelines }^{32}\end{array}$ & Trigger finger & \multicolumn{2}{|c|}{$\begin{array}{l}\text { A maioria dos casos pode ser abordada de forma conservadora (com } \\
\text { repouso, tala, AINE, infiltração de corticosteróide com anestésico). A } \\
\text { infiltração de corticosteróide com anestésico é mais efectiva do que } \\
\text { o anestésico isolado. Se for necessária força externa para estender o } \\
\text { dedo ou se não for possível estender o dedo deve recorrer-se a cirurgia. }\end{array}$} & \\
\hline
\end{tabular}




\begin{tabular}{|c|c|c|c|c|}
\hline $\begin{array}{l}\text { Tipo de artigo } \\
\text { Autor e ano }\end{array}$ & Título & Intervenções & Resultados & $\begin{array}{l}\text { NE/ } \\
\text { /FR }\end{array}$ \\
\hline $\begin{array}{l}\text { ECA } \\
\text { Baumgarten } \\
2007^{33}\end{array}$ & $\begin{array}{l}\text { Corticosteroid injection in diabetic } \\
\text { patients with trigger finger. A } \\
\text { prospective, randomized, } \\
\text { controlled double-blinded study }\end{array}$ & $\begin{array}{l}1 \text { ECA duplamente cego }(\mathrm{n}=35) \\
\text { em diabéticos. } \\
\text { Betametasona } 1 \mathrm{ml}+\text { lidocaína } \\
1 \% 0,5 \mathrm{ml} \text { versus placebo. } \\
\text { Estudo aberto: eficácia das } \\
\text { infiltrações nos diabéticos versus } \\
\text { não diabéticos. }\end{array}$ & $\begin{array}{l}\text { Não houve diferenças significativas } \\
\text { entre grupos na taxa de cirurgia } \\
\text { ou no alívio dos sintomas. } \\
\text { Após a 1.a infiltração não houve } \\
\text { diferenças significativas entre } \\
\text { grupos mas após a } 2 \text {. }^{\text {a injecção as }} \\
\text { infiltrações foram significativamente } \\
\text { mais eficazes nos não diabéticos. }\end{array}$ & 1 \\
\hline
\end{tabular}

\section{QUADRO VI. Resultados sobre a efectividade da infiltração de corticosteróide no ombro doloroso.}

\begin{tabular}{|c|c|c|c|c|}
\hline $\begin{array}{l}\text { Tipo de artigo } \\
\text { Autor e ano }\end{array}$ & Título & Intervenções & Resultados & $\begin{array}{l}\mathrm{NE} / \\
\text { /FR }\end{array}$ \\
\hline $\begin{array}{l}\text { Meta-análise } \\
\text { Arroll } 2005^{34}\end{array}$ & $\begin{array}{l}\text { Corticosteroid injections for } \\
\text { painful shoulder: a meta-analysis }\end{array}$ & $\begin{array}{l}7 \text { ECA infiltração subacromial } \\
\text { versus placebo; } 3 \text { ECA infiltração } \\
\text { subacromial versus AINE. }\end{array}$ & $\begin{array}{l}\text { Concluiu que as infiltrações de } \\
\text { corticosteróides são eficazes para } \\
\text { melhorar a tendinite da coifa até } \\
\text { aos nove meses e que é provável } \\
\text { que sejam mais eficazes que os AINE. }\end{array}$ & 1 \\
\hline $\begin{array}{l}\text { RS } \\
\text { Johansson } \\
2002^{35}\end{array}$ & $\begin{array}{l}\text { A combination of systematic } \\
\text { review and clinicians' beliefs in } \\
\text { interventions for subacromial pain }\end{array}$ & $\begin{array}{l}12 \text { Ensaios clínicos ( } 5 \text { ECA de } \\
\text { nível 1e a maioria dos outros de } \\
\text { nível 2). }\end{array}$ & $\begin{array}{l}\text { A injecção de corticosteróides foi } \\
\text { o único tratamento para a dor } \\
\text { subacromial que teve evidência } \\
\text { definitiva de eficácia a curto prazo } \\
\text { (< } 6 \text { semanas) e provavelmente } \\
\text { será eficaz a longo prazo. }\end{array}$ & 1 \\
\hline $\begin{array}{l}\text { RS } \\
\text { Buchbinder } \\
2003^{36}\end{array}$ & $\begin{array}{l}\text { Corticosteroid injections for } \\
\text { shoulder pain }\end{array}$ & $\begin{array}{l}7 \text { ECA infiltração subacromial } \\
\text { versus placebo; } 3 \text { ECA infiltração } \\
\text { subacromial versus AINE; } 1 \text { ECA } \\
\text { infiltração subacromial e AINE } \\
\text { versus AINE. }\end{array}$ & $\begin{array}{l}\text { A infiltração subacromial de } \\
\text { corticosteróide demonstrou ter, } \\
\text { em alguns estudos, um benefício } \\
\text { reduzido em relação ao placebo. } \\
\text { Com base nos resultados } \\
\text { combinados de três ensaios, não } \\
\text { foi demonstrado benefício da } \\
\text { infiltração de corticosteróides em } \\
\text { relação aos AINE. }\end{array}$ & 1 \\
\hline
\end{tabular}

ticosteróide na bursite anserina, nenhum dos artigos obtidos na pesquisa cumpriu os critérios de elegibilidade. São necessários estudos de boa qualidade metodológica.

\section{CONCLUSÃO}

Com base nos resultados desta revisão, os autores fazem as seguintes sugestões de actuação para o trata- mento pelo médico de família de utentes com doenças reumáticas peri-articulares numa unidade de saúde de CSP em que esteja disponível a opção terapêutica infiltração peri-articular de corticosteróide:

1. No doente com síndroma do túnel cárpico de gravidade ligeira a moderada, após modificação de actividade, ponderar a infiltração de corticoste- 


\section{QUADRO VI. (continuação)}

\begin{tabular}{|c|c|c|c|c|}
\hline $\begin{array}{l}\text { Tipo de artigo } \\
\text { Autor e ano }\end{array}$ & Título & Intervenções & Resultados & $\begin{array}{l}\text { NE/ } \\
\text { /FR }\end{array}$ \\
\hline $\begin{array}{l}\text { RS } \\
\text { Murphy } 2010^{37}\end{array}$ & Shoulder pain & $\begin{array}{l}5 \text { ECA ( } n=295) \text { infiltração de } \\
\text { corticosteróide e anestésico } \\
\text { versus anestésico; } 1 \text { ECA }(n=207) \\
\text { infiltração de corticosteróide } \\
\text { versus fisioterapia. }\end{array}$ & $\begin{array}{l}\text { Não foi encontrada informação } \\
\text { directa sobre se as infiltrações } \\
\text { sub-acromiais de corticosteróide } \\
\text { seriam melhores do que ausência } \\
\text { de tratamento activo. } \\
\text { Não foram encontradas diferenças } \\
\text { significativas entre a infiltração e } \\
\text { a fisioterapia na incapacidade aos } \\
6 \text { meses. A infiltração comparada } \\
\text { com a fisioterapia pode aumentar } \\
\text { a necessidade de repetir consultas } \\
\text { ou intervenções ( } 57 \text { versus } 40 \% \text { ). }\end{array}$ & 1 \\
\hline $\begin{array}{l}\text { RS/Meta-análise } \\
\text { Coombes } 2010^{21}\end{array}$ & $\begin{array}{l}\text { Efficacy and safety of } \\
\text { corticosteroid injections and other } \\
\text { injections for management of } \\
\text { tendinopathy: a systematic review } \\
\text { of randomised controlled trials }\end{array}$ & 10 ECA; 14 análises $(n=780)$ & $\begin{array}{l}\text { Existe evidência inconsistente } \\
\text { sobre a eficácia da infiltração de } \\
\text { corticosteróide a curto-prazo. No } \\
\text { entanto, a análise de um subgrupo } \\
\text { de três estudos mostrou um efeito } \\
\text { benéfico da infiltração com } \\
\text { corticosteróides em comparação } \\
\text { com o placebo. Não houve } \\
\text { diferença na eficácia em todos os } \\
\text { estudos em que foram prescritos } \\
\text { AlNEs orais. Os estudos que } \\
\text { compararam a injecção de } \\
\text { corticosteróides com a fisioterapia } \\
\text { não demonstraram diferenças na } \\
\text { dor ou na função a médio e longo } \\
\text { prazo. Contudo num dos estudos } \\
\text { mais doentes relataram melhoria } \\
\text { geral seis semanas após a injecção } \\
\text { de corticosteróide. }\end{array}$ & 1 \\
\hline $\begin{array}{l}\text { Normas da } \\
\text { melhor prática } \\
\text { EBM Guidelines } \\
2009^{38}\end{array}$ & Biceps tendon disorders & \multicolumn{2}{|c|}{$\begin{array}{l}\text { O tratamento da tendinite bicipital inclui repouso, AINE, injecção de } \\
\text { corticosteróide na área dolorosa e fisioterapia. }\end{array}$} & nd* \\
\hline \multirow{5}{*}{$\begin{array}{l}\text { Normas da } \\
\text { melhor prática } \\
\text { EBM Guidelines } \\
2009^{39}\end{array}$} & \multirow{5}{*}{$\begin{array}{l}\text { Disorders of the rotator cuff of } \\
\text { the shoulder }\end{array}$} & \multicolumn{2}{|c|}{ A 1. ${ }^{\text {a }}$ abordagem consiste no repouso e AINEs. } & nd* \\
\hline & & \multicolumn{2}{|c|}{ 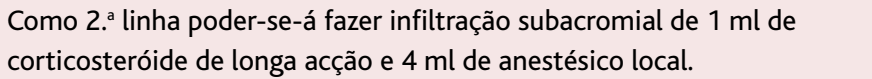 } & B \\
\hline & & \multicolumn{2}{|c|}{ A infiltração poderá ser repetida 1-3 vezes. } & nd* \\
\hline & & \multicolumn{2}{|c|}{ Nas situações prolongadas a fisioterapia poderá ser benéfica. } & C \\
\hline & & \multicolumn{2}{|c|}{$\begin{array}{l}\text { Na tendinite cálcica a infiltração subacromial de corticosteróide pode } \\
\text { ajudar e na bursite ou periartrite cálcica a infiltração é considerada } \\
\text { de 1. linha. }\end{array}$} & nd* \\
\hline
\end{tabular}




\begin{tabular}{|c|c|c|c|}
\hline $\begin{array}{l}\text { Tipo de artigo } \\
\text { Autor e ano }\end{array}$ & Título & Intervenções & $\begin{array}{l}\mathrm{NE} / \\
\text { /FR }\end{array}$ \\
\hline $\begin{array}{l}\text { Normas da } \\
\text { melhor prática } \\
\text { EBM Guidelines } \\
2009^{39}\end{array}$ & $\begin{array}{l}\text { Disorders of the rotator cuff of } \\
\text { the shoulder }\end{array}$ & $\begin{array}{l}\text { A 1. abordagem consiste no repouso e AINEs. } \\
\text { Como 2. linha poder-se-á fazer infiltração subacromial de } 1 \mathrm{ml} \text { de } \\
\text { corticosteróide de longa acção e } 4 \mathrm{ml} \text { de anestésico local. } \\
\text { A infiltração poderá ser repetida } 1-3 \text { vezes. } \\
\text { Nas situações prolongadas a fisioterapia poderá ser benéfica. } \\
\text { Na tendinite cálcica a infiltração subacromial de corticosteróide pode } \\
\text { ajudar e na bursite ou periartrite cálcica a infiltração é considerada } \\
\text { de 1. linha. }\end{array}$ & $\begin{array}{c}\mathrm{nd}^{*} \\
\mathrm{~B} \\
\mathrm{nd}^{*} \\
\mathrm{C} \\
\mathrm{nd}^{*}\end{array}$ \\
\hline $\begin{array}{l}\text { Normas da } \\
\text { melhor prática } \\
\text { Work Loss Data } \\
\text { Institute } 2008^{40}\end{array}$ & Shoulder (acute \& chronic) & $\begin{array}{l}\text { Na primeira consulta deve ser prescrito repouso, alteração da } \\
\text { actividade e analgesia por via oral (acetaminofeno e/ou AINEs). Se } \\
\text { não houver melhoria significativa deve-se propor fisioterapia. O } \\
\text { controlo adicional da dor pode ser conseguido através da infiltração } \\
\text { subacromial com corticosteróide. } \\
\text { Pode-se repetir a infiltração no máximo três vezes em intervalos não } \\
\text { inferiores a três semanas. } \\
\text { A infiltração deve ser evitada em doentes com menos de } 30 \text { anos de idade. }\end{array}$ & nd* \\
\hline $\begin{array}{l}\text { Normas da } \\
\text { melhor prática } \\
\text { EBM Guidelines } \\
2011^{41}\end{array}$ & $\begin{array}{l}\text { Local corticosteroid injections in } \\
\text { soft tissues and joints }\end{array}$ & $\begin{array}{l}\text { A infiltração subacromial de corticosteróide parece proporcionar, a } \\
\text { curto prazo, melhoria da dor e da amplitude dos movimentos. É } \\
\text { recomendada quando há restrição dos movimentos, bursite } \\
\text { subacromial ou inflamação do tendão suprasespinhoso. }\end{array}$ & B \\
\hline
\end{tabular}

*Não definido

\section{QUADRO VII. Resultados sobre a efectividade da infiltração de corticosteróide na bursite trocantérica.}

\begin{tabular}{|c|c|c|c|}
\hline $\begin{array}{l}\text { Tipo de artigo } \\
\text { Autor e ano }\end{array}$ & Título & Resultados & $\begin{array}{l}\mathrm{NE} / \\
\text { /FR }\end{array}$ \\
\hline $\begin{array}{l}\text { Normas da } \\
\text { melhor prática } \\
\text { EBM Gulideines } \\
2010^{42}\end{array}$ & Trochanteric pain & $\begin{array}{l}\text { O tratamento de eleição da bursite trocantérica é uma injecção de } \\
\text { corticosteróide ( } 1 \mathrm{ml}) \text { e anestésico local (1-2 ml lidocaína a 1\%) no } \\
\text { sítio mais doloroso. } \\
\text { Se a primeira infiltração não aliviar a dor, o tratamento pode ser } \\
\text { repetido após algumas semanas. }\end{array}$ & $C$ \\
\hline $\begin{array}{l}\text { Normas da } \\
\text { melhor prática } \\
\text { EBM Gulideines } \\
2011^{41}\end{array}$ & $\begin{array}{l}\text { Local corticosteroid injections in } \\
\text { soft tissues and joints }\end{array}$ & $\begin{array}{l}\text { A infiltração com corticosteróide e lidocaína pode ser um tratamento } \\
\text { efectivo para a dor da bursite trocantérica. }\end{array}$ & $C$ \\
\hline
\end{tabular}

róide antes de referenciar o doente para cirurgia (não é claro se a cirurgia é mais efectiva do que a infiltração mas é mais provável que a cirurgia cause efeitos adversos).

2. No doente com epicondilite, ponderar infiltração de corticosteróide se a sintomatologia for grave e não melhorar após quatro semanas de tratamento conservador (melhora os sintomas a curto prazo mas os efeitos revertem com o tempo); se necessário repetir a infiltração algumas semanas após a primeira.

3. No doente com tenossinovite de De Quervain, 
pode utilizar-se como primeira linha a infiltração de corticosteróide, para além das alterações de adaptação ergonómica e do uso de tala.

4. No doente com dedo em gatilho, considerar como primeira linha a terapêutica com infiltração de corticosteróide; se necessário pode repetir-se a infiltração após um intervalo de pelo menos quatro semanas.

5. No doente com dor no ombro, ponderar a infiltração de corticosteróide. Considerar como primeira linha se os sintomas limitam os exercícios de reabilitação e na bursite cálcica.

6. No doente com bursite trocantérica, propor como tratamento de primeira linha a infiltração de corticosteróide. Se a primeira infiltração não aliviar a dor, o tratamento pode ser repetido.

7. Em relação à bursite anserina, há escassa evidência e resultados discordantes sobre efectividade da infiltração de corticosteróide pelo que se sugere avaliar caso a caso a relação risco-benefício do procedimento.

\section{REFERÊNCIAS BIBLIOGRÁFICAS}

1. Direcção Geral de Saúde. Programa Nacional contra as Doenças Reumáticas; Despacho Ministerial de 26/03/2004. Lisboa; DGS; 2004.

2. Lucas R, Monjardino MT. O estado da reumatologia em Portugal. Observatório Nacional das Doenças Reumáticas - Programa Nacional Contra as Doenças Reumáticas. Lisboa: 2010. p. 83-91.

3. Dooley P, Martin R. Corticosteroid injections and arthrocentesis. Can Fam Physician 2002 Feb; 48: 285-92.

4. Magaña Loarte JE, Pérez Franco J, Sánchez Sánchez G. Es factible la terapia con inflitraciones locales en las consultas de atención primaria? Aten Primaria 1999 Ene; 23 (1): 4-7.

5. Ebell MH, Siwek J, Weiss BD, Woolf SH, Susman J, Ewigman B, et al. Strength of Recommendation Taxonomy (SORT): a patient-centered approach to grading evidence in the medical literature. Am Fam Physician 2004 Feb 1; 69 (3): 548-56.

6. Roque A, Bugalho A, Carneiro AV. Manual de elaboração, disseminação e implementação de Normas de Orientação Clínica. Lisboa: Centro de Estudos de Medicina Baseada na Evidência - Faculdade de Medicina de Lisboa; 2007.

7. Gerritsen AA, de Krom MC, Struijs MA, Scholten RJ, de Vet HC, Bouter LM. Conservative treatment options for carpal tunnel syndrome: a systematic review of randomised controlled trials. J Neurol 2002 Mar; 249 (3): 272-80.

8. Coates VH, Turkelson CM, Chapell R, BrueningW, Mitchell MD, Reston JT, et alDiagnosis and Treatment of Worker Related Musculoskeletal Disorders of the Upper Extremity. Evidence Report/Technology Assessment Number 62. AHRQ Publication No. 02-E038. Rockville, MD: Agency for Healthcare Research and Quality; 2002.

9. Piazzini DB, Aprile I, Ferrara PE, Bertolini C, Tonali P, Maggi L, et al. A systematic review of conservative treatment of carpal tunnel syndrome. Clinical Rehabilitation 2007 Apr; 21 (4): 299-314.

10. Marshall S, Tardif G, Ashworth NL. Local corticosteroid injection for carpal tunnel syndrome. Cochrane Database Syst Rev 2007 Apr 18; (2): CD001554.
11. Ashworth N. Carpal tunnel syndrome. Clin Evid (Online). 2010 Mar 23; 2010. Disponível em:http://www.ncbi.nlm.nih.gov/pmc/articles/PMC2907614/ [acedido em 20/03/2011].

12. Peters-Veluthamaningal C, Winters JC, Groenier KH, Meyboom-de-Jong B. Randomised controlled trial of local corticosteroid injections for carpal tunnel syndrome in general practice. BMC Fam Pract 2010 Jul 29; 11: 54.

13. Verdugo RJ, Salinas RA, Castillo JL, Cea JG. Surgical versus non-surgical treatment for carpal tunnel syndrome. Cochrane Database Syst Rev 2008 Oct 8; CD001552.

14. Work Loss Data Institute. Carpal tunnel syndrome (acute \& chronic). 2008. Disponível em: http://www.essentialevidenceplus.com [acedido em 20/03/2011].

15. Assmus H, Antoniadis G, Bischoff C, Haussmann P, Martini AK, Mascharka Z, et al. Diagnostik undTherapie des Karpaltunnelsyndroms — Leitlinie der Deutschen Gesellschaften fur Handchirurgie, Neurochirurgie, Neurologie, Orthopadie unter Mitbeteiligung der Gesellschaften fur Unfallchirurgie, Klinische Neurophysiologie und Funktionelle Bildgebung sowie Plastiche, Rekonstruktive und Asthetische Chirurgie. Handchir Mikrochir Plast Chir 2007 Aug; 39(4): 276-88.

16. American Academy of Orthopaedic Surgeons. AAOS Clinical Practice Guideline Treatment of Carpal Tunnel Syndrome. Rosemont, IL:American Academy of Orthopaedic Surgeons; 2008. Disponível em:http://www.aaos.org/research/guidelines/guide.asp [acedido em 20/03/2011].

17. EBM Guidelines. Carpal tunnel syndrome. 2009. Disponível em: http://www.essentialevidenceplus.com [acedido em 20/03/2011].

18. Smidt N, Assendelft WJ, van der Windt D A, Hay EM, Buchbinder R, Bouter LM. Corticosteroid injections for lateral epicondylitis: a systematic review. Pain 2002 Mar; 96 (1-2): 23-40.

19. Buchbinder R, Green SE, Struijs P. Tennis elbow. Clin Evid (Online). 2008 May 28; 2008. Disponível em: http://www.ncbi.nlm.nih.gov/pmc/articles/ PMC2907994/ [acedido em 21/03/2011]

20. Barr S, Cerisola FL, Blanchard V. Effectiveness of corticosteroid injections compared with physiotherapeutic interventions for lateral epicondylitis: a systematic review. Physiotherapy 2009 Dec; 95 (4): 251-65.

21. Coombes BK, Bisset L, Vicenzino B. Efficacy and safety of corticosteroid injections and other injections for management of tendinopathy: a systematic review of randomised controlled trials. Lancet 2010 Nov 20; 376 (9754): 1751-67.

22. Lin YC, TuYK, Chen SS, Lin IL, Chen SC, Guo HR. Comparison between botulinum toxin and corticosteroid injection in the treatment of acute and subacute tennis elbow: a prospective, randomized, double-blind, active drug-controlled pilot study. Am J Phys Med Rehabil 2010 Aug; 89 (8): 653-9.

23. Varonen $\mathrm{H}$. Lateral and medial epicondylitis of elbow. EBM Guidelines; 2008. Disponível em: http://www.essentialevidenceplus.com [acedido em 20/03/2011].

24. Work Loss Data Institute. Elbow (acute \& chronic). 2008. Disponível em: http://www.essentialevidenceplus.com [acedido em 20/03/2011].

25. American College of Occupational and Environmental Medicine (ACOEM). Elbow disorders. Elk Grove Village, IL:American College of Occupational and Environmental Medicine (ACOEM); 2007.

26. Peters-Veluthamaningal $C$, van der Windt DA, Winters JC, Meyboom-de Jong B. Corticosteroid injection for de Quervain's tenosynovitis. Cochrane Database Syst Rev 2009 Jul 8; (3): CD005616.

27. Peters-Veluthamaningal C, Winters JC, Groenier KH, Meyboom-dejong B. Randomised controlled trial of local corticosteroid injections for de Quervain's tenosynovitis in general practice. BMC Musculoskelet Disord 2009 Oct 27; 10: 131.

28. Viikari-Juntura E. De Quervain's disease and other tendinitides of the wrist and forearm. EBM Guidelines. 2009. Disponível em: http://www.essentialevidenceplus.com [acedido em 20/03/2011].

29. Peters-Veluthamaningal C, van der Windt DA, Winters JC, Meyboom-de-Jong 
B. Corticosteroid injection for trigger finger in adults. Cochrane Database Syst Rev 2009 Jan 21; (1): CD005617.

30. Fleisch SB, Spindler KP, Lee DH. Corticosteroid injections in the treatment of trigger finger: a level I and II systematic review. J Am Acad Orthop Surg 2007 Mar; 15 (3): 166-71.

31. Peters-Veluthamaningal C, Winters JC, Groenier KH, Jong BM. Corticosteroid injections effective for trigger finger in adults in general practice: a double-blinded randomised placebo controlled trial. Ann Rheum Dis 2008 Sep; 67 (9): 1262-6.

32. EBM Guidelines. Trigger finger. 2010. Disponível em: http://www.essentialevidenceplus.com/content/ebmg_ebm/1053 [acedido em 20/03/2011].

33. Baumgarten KM, Gerlach D, Boyer MI. Corticosteroid injection in diabetic patients with trigger finger: a prospective, randomized, controlled double-blinded study. J Bone Joint Surg Am 2007 Dec; 89 (12): 2604-11.

34. Arroll B, Goodyear-Smith F. Corticosteroid injections for painful shoulder: a meta-analysis. Br J Gen Pract 2005 Mar; 55 (512): 224-8.

35. Johansson K, Oberg B, Adolfsson L, Foldevi M. A combination of systematic review and clinicians' beliefs in interventions for subacromial pain. $\mathrm{Br} J$ Gen Pract 2002 Feb; 52 (475): 145-52.

36. Buchbinder R, Green S, Youd JM. Corticosteroid injections for shoulder pain. Cochrane Database Syst Rev 2003; 1: CD004016.

37. Murphy RJ, Carr AJ. Shoulder pain. Clin Evid (Online); 2010 Jul 22; 20110. Disponível em:http://www.ncbi.nlm.nih.gov/pmc/articles/PMC3217726/ [acedido em 21/03/2011].

38. Vastamäki M. Biceps tendon disorders. EBM Guidelines; 2009. Disponível em: http://ebmg.onlinelibrary.wiley.com [acedido em 20/03/2011].
39. Vastamäki M. Disorders of the rotator cuff of the shoulder. EBM Guidelines; 2009. Disponível em: http://ebmg.onlinelibrary.wiley.com [acedido em 20/03/2011].

40. Work Loss Data Institute. Shoulder (acute \& chronic). 2008. Disponível em: http://www.essentialevidenceplus.com [acedido em 20/03/2011].

41. Kunnamo I. Local corticosteroid injections in soft tissues and joints. 2011. Disponível em: http://www.essentialevidenceplus.com [acedido em 20/03/2011].

42. Pienimäki T. Trochanteric pain. EBM Guidelines; 2010. Disponível em: http://ebmg.onlinelibrary.wiley.com [acedido em 20/03/2011].

\section{CONFLITOS DE INTERESSES DOS AUTORES}

Os autores declaram não possuir qualquer tipo de conflito de interesses.

\author{
ENDEREÇO PARA CORRESPONDÊNCIA \\ Ana Rita Ferreira \\ Largo Dom Constantino de Bragança $\mathrm{n}^{\circ} 11^{\circ} \mathrm{B}$ \\ 2720-162 Reboleira/Amadora \\ anarita.rferreira@gmail.com \\ Eunice Carrapiço \\ Rua Gama Pinto ${ }^{\circ} 114^{\circ} \mathrm{B}$ \\ 2675-648 Odivelas (Colinas do Cruzeiro) \\ eunicecarrapico@gmail.com
}

Recebido em 28/10/2012

Aceite para publicação em 28/12/2012

\section{ABSTRACT}

\section{CORTICOSTEROID INJECTIONS FOR PERIARTICULAR RHEUMATIC DISEASES: AN EVIDENCE-BASED REVIEW}

Introduction: Periarticular rheumatic diseases are common and cause a significant economic and social burden. They are frequent causes of visits to family doctors. Corticosteroid injections are important adjuvants in the treatment of rheumatic diseases. They can be performed by doctors with the necessary skills. The aim of this paper is to review the evidence for the effectiveness of corticosteroid injections in seven common periarticular rheumatic diseases.

Methodology: We selected seven common periarticular rheumatic diseases for review: carpal tunnel syndrome, epicondylitis, De Quervain's disease, trigger finger, shoulder pain, trochanteric bursitis, and anserine bursitis. A systematic search for systematic reviews, randomized controlled trials and clinical guidelines was conducted for each condition. The searches were done in March 2011 for papers with the following eligibility criteria: (1) a population of patients diagnosed with one of the selected rheumatic diseases, (2) an intervention with corticosteroid injection versus no treatment, placebo or other treatments, (3) patient-oriented outcomes regarding effectiveness, (4) publication in or after 2000. We excluded studies not available in full text.

Results: Thirty-six studies were included in this review. For carpal tunnel syndrome, corticosteroid injection was found to be more effective than placebo. Corticosteroid infiltration is also beneficial in reducing symptoms in the short-term for epicondylitis. In de Quervain's disease, corticosteroid infiltration is recommended as the initial treatment. For trigger finger, infiltration is effective and safe compared to placebo. Is was not possible to make clear recommendations for corticosteroid injections for shoulder pain. For trochanteric bursitis, there is weak evidence for the use of infiltration. No eligible studies were found to evaluate the effectiveness of corticosteroid injection for anserine bursitis.

Conclusion: The infiltration of corticosteroids steroids proved effective in treating four of the seven periarticular rheumatic diseases chosen for this review. There are considerable differences in the magnitude and duration of therapeutic effects in different conditions which makes it impossible to generalize regarding the effectiveness of corticosteroid injection for all periarticular rheumatic diseases.

Keywords: Injections; Corticosteroid; Effectiveness; Rheumatic Disease; Periarthritis. 